\title{
Visualizing excited state dynamics of conjugated molecules trough femtosecond stimulated Raman scattering
}

\author{
Giovanni Batignanil, ${ }^{1,}$,Emanuele Pontecorvo ${ }^{1}$, Carino Ferrante ${ }^{1,2}$, Massimiliano Aschi ${ }^{3}$, \\ Christopher G. Elles ${ }^{4}$, Tullio Scopigno ${ }^{1,2}$ \\ ${ }^{1}$ Dipartimento di Fisica, Università di Roma "La Sapienza", Roma, 00185, Italy. \\ ${ }^{2}$ Istituto Italiano di Tecnologia, Center for Life Nano Science@Sapienza, Roma, 00161, Italy \\ ${ }^{3}$ Dipartimento di Scienze Fisiche e Chimiche, Università degli studi dell'Aquila, L'Aquila, 67100, I \\ Italy \\ ${ }^{4}$ Department of Chemistry, University of Kansas, Lawrence, Kansas, 66045, USA.
}

\begin{abstract}
The reaction pathway in the photoexcited model compound 2methyl-5-phenylthiophene has been unravelled by Femtosecond Stimulated Raman Scattering and quantum chemical calculations. The excited state dynamics, including structural rearrangement, vibrational cooling and intersystem-crossing, will be presented.
\end{abstract}

\section{Background and significance}

There is continuing interest in the photo-physical properties of conjugated organic polymers, as there are significant processing advantages for using these materials to manufacture organic optoelectronic devices, such as photochromic molecular switches, solar energy conversion devices and light-emitting diodes. Accessing and manipulating the photochemical properties of these versatile materials requires a fundamental understanding of the reaction pathway of their building blocks upon an ultrashort optical excitation. In fact, although the performance of such materials ultimately depends on the fundamental properties of the constituent molecules, the dynamics of large polymeric systems are often difficult to discern due to structural heterogeneities.

A key question to be answered is the role of structural recombination following photoexcitation. For example, ultrafast geometrical rearrangement affects the initial stages of charge separation and recombination and also provides valuable clues about the chargetransport material properties.

In this respect, spontaneous Raman (SR) represents a powerful tool to probe the geometrical configuration of molecular and solid-state systems. Taking advantage of its inherent sensitivity to the vibrational properties, it allows to extract structural and dynamical information. However, when it is used as a probe in time-resolved experiments to address transient phases, it has a critical shortcoming. Specifically, it is subject to the time-energy resolution restriction dictated by the time-bandwidth product of the light pulse,

* Corresponding author: giovanni.batignani@uniroma1.it 
which is limited by $\Delta \mathrm{E} \cdot \Delta \mathrm{t} \geq 15 \mathrm{~cm}^{-1}$ ps, preventing SR to access picosecond and subpicosecond dynamics.

\section{Methods and results}

Femtosecond Stimulated Raman Scattering (FSRS) [1] is a recently developed technique allowing for vibrational spectroscopy with sub-ps time precision. In FSRS, a femtosecond actinic pulse (AP) initiates the photochemistry of interest. The system is subsequently interrogated by a pair of overlapped pulses: the joint presence of a broadband ultrashort probe pulse (PP) and a narrowband picosecond Raman pulse (RP) induces vibrational coherences which are read out as heterodyne coherent Raman signals free of fluorescence background, with simultaneous high spectral resolution and time precision [2].

Here we reveal the full reaction pathway of photoexcited 2-methyl-5-phenylthiophene (MPT), a model compound for conjugated organic polymers. This is achieved by developing a FSRS setup with broad tunability of the Raman pulse [3-5] that, by matching the electronic transitions corresponding to excited state absorption (ESA) and stimulated emission (SE) measured in the transient absorption (TA) spectrum (Fig. 1a), allows us to selectively isolate dynamics from singlet and triplet transient species.

A magnetic transition (intersystem crossing) to the triplet state is experimentally monitored by tuning the Raman pulse to $366 \mathrm{~nm}$. Particularly, this wavelength is initially resonant with the ESA and SE from the excited singlet state $\left(\mathrm{S}_{1}\right)$, while it matches the ESA transition of the triplet state $\left(T_{1}\right)$ for times longer than $100 \mathrm{ps}$. The simultaneous decay of the $\mathrm{S}_{1}$ Raman bands and the build-up of the T1 modes indicate an intersystem crossing on a $\tau_{3} \approx 135 \mathrm{ps}$ timescale. In striking contrast, tuning the RP wavelength at $480 \mathrm{~nm}$, i.e. solely resonant with the singlet ESA, enables for selective tracking of the structural rearrangement on the $\mathrm{S}_{1}$ surface.

In order to rationalize the experimental results, time-dependent (TD) density functional theory (DFT) calculations have been performed. Specifically, we evaluated the potential energy surfaces (PES) reported in Fig. 1b, the corresponding normal modes and the diabatic transition probabilities for the ISC between singlet and triplet excited states. A comparison of the FSRS spectra and TD-DFT results indicates that the relaxation process is driven by two internal coordinates: the dihedral angle between phenyl and thiophene rings and the distance $\mathrm{d}_{\mathrm{C}-\mathrm{S}}$ between the carbon (connected to phenyl) and the sulfur atoms in the thiophene.

In particular, the evaluation of calculated vibrational frequencies on the S1 state is in excellent agreement with the FSRS spectra, with Raman band blue-shifts observed by FSRS within the first 10 ps pointing to a vibrational cooling process. Notably, the normal modes computed along the PES for an open-thiophene-ring configuration are not compatible with the Raman band positions at times longer than $\tau_{2} \approx 10 \mathrm{ps}$, ruling out slow structural rearrangement, which on the contrary occurs on a faster timescale $\left(\tau_{1} \approx 1.5 \mathrm{ps}\right)$. This latter involves a planarization of the system, with the wavepacket evolving toward the $\mathrm{S}_{1}$ PES local minimum (close to the Franck-Condon region) and is accompanied by a spread of the probability distribution along the $\mathrm{d}_{\mathrm{C}-\mathrm{S}}$ coordinate.

\section{Conclusions}

Taken together, our results elucidate the specific mechanism how different concurring processes drive in an orchestrated way the MPT photo-reaction to the relaxed triplet state. Specifically, we revealed an initial rapid structural relaxation $\left(\tau_{1} \approx 1.5 \mathrm{ps}\right)$, followed by thermalization on the way to the S1 PES local minimum ( $\left.\tau_{2} \approx 10 \mathrm{ps}\right)$. Also, we identified an 
intersystem crossing to the triplet state, which involves a slow opening of the thiophene ring through a cleavage of the carbon-sulfur bond $\left(\tau_{3} \approx 135 \mathrm{ps}\right)$, required to access the intersystem crossing curve [6].

(a)

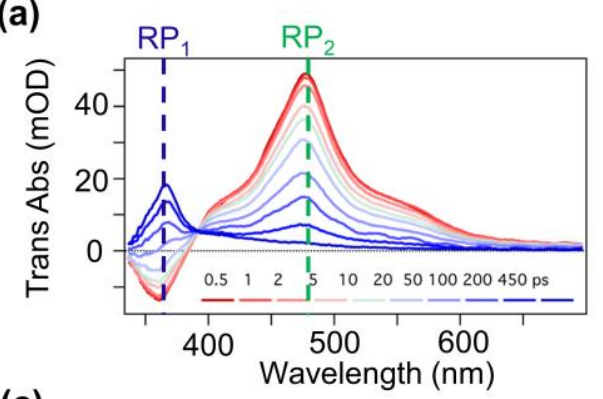

(b)

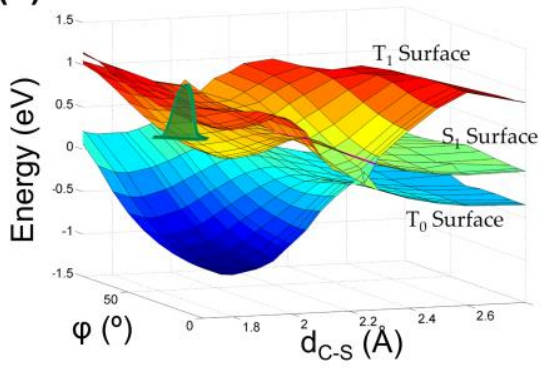

(c)

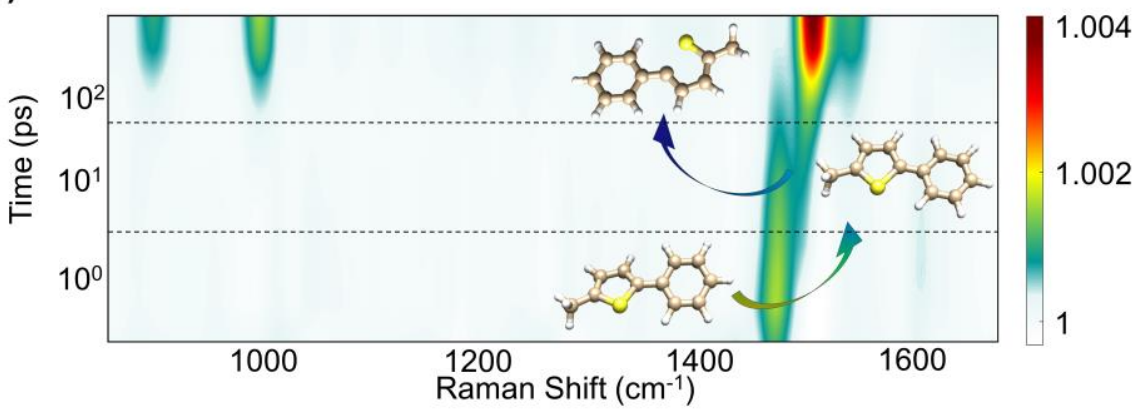

Fig. 1. (a) Transient absorption spectrum of MPT following a $266 \mathrm{~nm}$ excitation to the $\mathrm{S}_{1}$ potential energy surface. The dashed lines indicate the Raman pulse wavelengths used in the FSRS experiments. (b) MPT excited potential energy surfaces computed by TD-DFT with B3LYP functional and the $6-31+\mathrm{g}^{* *}$ basis set along the reaction coordinates $\varphi$ and $d_{C-s .}$ (c) FSRS spectrum of MPT upon photo-excitation. After an ultrafast planarization and cooling of the system promoted on the excited $\mathrm{S}_{1}$ state, the slow opening of the thiophene ring triggers an intersystem crossing to the triplet state.

\section{References}

1. D. W. McCamant, P. Kukura, S. Yoon, and R. A. Mathies, Rev. Sci. Instrum. 75, 4971-4980 (2004).

2. G. Fumero, G. Batignani, K. E. Dorfman, S. Mukamel and T. Scopigno, Chem. Phys. Chem. 16, 3438-43 (2015).

3. G. Batignani, D. Bossini, N. Di Palo, C. Ferrante, E. Pontecorvo, G. Cerullo, A. Kimel and T. Scopigno, Nature Photon. 9, 506 (2015).

4. G. Batignani, E. Pontecorvo, G. Giovannetti, C. Ferrante, G. Fumero and T. Scopigno, Sci. Rep. 6, 18445 (2016).

5. G. Batignani, G. Fumero, S. Mukamel, T. Scopigno, Phys Chem Chem Phys. 17(16), 10454 (2015).

6. [4] G. Batignani, E. Pontecorvo, C. Ferrante, M. Aschi, C. G. Elles and T. Scopigno, J. Phys. Chem. Lett. 4,7(15), 2981 (2016). 